\title{
Comunidades receptoras y percepciones: un estudio sobre turismo y sexualidad
}

DOI: 10.22403/UQROOMX/TYP05/11

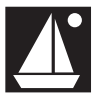

\section{J. Carlos Monterrubio Cordero*}

RESUMEN

Como una investigación cualitativa sobre las percepciones de comunidades locales hacia el turismo gay y su comportamiento sexual, el presente artículo pretende contribuir al entendimiento de las dimensiones de los impactos del turismo y reconocer el valor de metodologías interpretativas para el estudio de temas delicados en la investigación turística.

Con base en una triangulación metodológica, la investigación fue realizada a través de entrevistas a profundidad, del análisis de contenido de sitios web y de conversaciones informales en un destino turístico de la costa del Pacífico de México. La muestra de informantes clave estuvo compuesta sobre todo por residentes locales y turistas gay; reunidos mediante un muestreo bola de nieve.

La investigación proporciona una idea general de las percepciones hacia el turismo gay y de los posibles impactos reales y potenciales de su comportamiento sexual en comunidades rurales en países en vías desarrollo. El estudio reveló que la presencia y el beneficio económico de visitantes gay han sido ampliamente reconocidos por la comunidad local y que el comportamiento sexual de dichos visitantes está generando problemas sociales, tales como la prostitución varonil y el uso de drogas.Asimismo, se encontró que esta conducta aliena a otras formas de turismo y a la comunidad local de espacios públicos de recreación.

Palabras | Investigación cualitativa, impactos del turismo, percepciones del

CLAVE turismo, turismo gay, turismo sexual. 


\section{Introducción}

Las relaciones entre el turismo y la sexualidad han captado el interés académico durante las últimas décadas, y los expertos han establecido la urgencia de identificar los impactos de este tipo de turismo (Hughes, 2006). Desde sus inicios, la mayoría de los estudios se ha concentrado en los aspectos comerciales de estas relaciones, haciendo a un lado otras dimensiones relevantes tales como sus efectos reales y potenciales en comunidades receptoras de turismo.Aunque determinadas investigaciones han abordado las repercusiones de los vínculos entre turismo y sexualidad (Leung, 2003), tanto sus dimensiones como las experiencias sexuales de los viajeros gay y sus consecuencias sobre las comunidades receptoras requieren mayor atención.

Como parte de una investigación exploratoria de carácter cualitativo, el presente artículo se propone contribuir al entendimiento de los efectos sociales del turismo, analizando la naturaleza del comportamiento sexual del turismo gay. También tiene como objetivo apoyar el reconocimiento del valor académico de metodologías cualitativas para el estudio de temas delicados en investigaciones turísticas. Por ello, se exponen brevemente algunas dimensiones de las secuelas de la interacción de turistas gay y los miembros de una comunidad local dentro de un contexto sexual, obtenidas a través de metodologías no positivistas.

En los resultados destaca que los residentes de la comunidad local han percibido claramente el incremento de visitantes gay durante los últimos años,y su importancia económica ha sido reconocida en la comunidad. Sin embargo, dentro de los asuntos más sobresalientes se encontró que existe actividad sexual entre visitantes gay en espacios públicos del destino turístico.También se observó la propagación del pago por servicios sexuales, de la prostitución de varones jóvenes de la localidad y del uso de drogas entre ellos.Se concluyó que el comportamiento sexual del turismo gay ha tenido otras implicaciones sociales $y$ económicas entre los miembros de la comunidad receptora.

\section{Antecedentes}

\section{Turismo y sexo}

Turismo y sexo han estado inextricablemente ligados desde los inicios de los viajes (McKercher y Bauer, 2003). Mientras que el sexo o el prospecto 
de encuentros sexuales pueden jugar un papel central en la decisión de viajar, en ocasiones la actividad sexual representa un elemento incidental en los viajes o, definitivamente, puede no jugar rol alguno en tal decisión. El sexo es una parte natural de la vida. Si los individuos participan en actividades sexuales en casa, sería lógico suponer que también lo harán durante sus viajes. De hecho, el turismo simplemente provee un escenario diferente (McKercher y Bauer, 2003: 4).

A la fecha, se ha realizado un número limitado de investigaciones acerca de las relaciones entre el turismo y el sexo, y lo que éstas conllevan. La mayoría de los estudios se han enfocado en el aspecto comercial del denominado turismo sexual, aunque algunos tratan otros temas relacionados. Por ejemplo, ciertos investigadores han dirigido su atención hacia la dinámica de las relaciones entre sexoservidoras y turistas en países como Tailandia (Cohen, 1982); otros han abordado el asunto de mujeres que viajan al Caribe en busca de relaciones sexuales o románticas con los llamados beach boys (Dahles, 1998; Jeffreys, 2003; Kempadoo, 1999), o a países africanos en busca de vacaciones sexuales (Nyanzi et al., 2005). La actividad sexual en relación con el consumo de alcohol y drogas entre estudiantes de universidad en destinos de playa también ha sido examinada (Josiam et al., 1998). De igual forma, varones heterosexuales y homosexuales (Clift y Wilkins, 1995; Seabrook, 200I) así como pederastas (Beddoe, 2003) cuyo único propósito de viaje es involucrarse en actividades sexuales en países destino, se han convertido en objeto de análisis. Otros asuntos como los relativos a la salud, derivados de la actividad sexual durante los viajes, particularmente aquellos asociados a las infecciones sexualmente trasmisibles, han atraído el interés académico (Agrusa, 2003; Clift y Carter, 2000; Hobson y Dietrich, 1994; Rogstad, 2004).

Los estudios han variado en cuanto a espacio y tiempo, pero aún hay varios aspectos olvidados; por ejemplo, a pesar de la importancia de la identificación de los impactos del turismo para propósitos de planeación y gestión turística (Wall y Mathieson, 2006), muy escasas investigaciones se han enfocado en los efectos de las dimensiones del turismo y el sexo en comunidades receptoras. El estudio realizado por Leung (2003) ha sido uno de los pocos que han intentado investigar esto, pero el trabajo se orienta más hacia los posibles impactos que el negocio sexual tiene en el desarrollo económico, social y sanitario.Asimismo, aunque las percepciones de residentes locales hacia las repercusiones del tu- 
rismo han sido frecuentemente tratadas, las percepciones de residentes hacia tipos específicos de turismo, incluyendo al gay y su comportamiento, han sido desatendidas por la academia.

\section{Turismo, sexo y viajeros gay}

La investigación respecto a viajeros gay y su comportamiento sexual también es limitada (Oppermann, 1999:4), por ello ha sido difícil generar extrapolaciones significativas de los resultados, sobre todo porque las circunstancias, muestras y metodologías de cada estudio han variado considerablemente. En términos generales, la investigación existente relacionada con el comportamiento sexual de viajeros gay se ha concentrado en el grado de dicha actividad sexual y el nivel de riesgo de infecciones sexuales (Clift y Forrest, 1999).

En la literatura revisada, muy pocas dimensiones de las relaciones entre turismo, sexo y diversidad sexual (viajeros gay) han sido examinadas. Uno de los primeros trabajos reportados es el llevado a cabo en Tailandia (Wilke y Kleiber, 1992, cit. en Clift y Wilkins, 1995), el cual tuvo como objetivo identificar qué hombres visitaban el país como "turistas sexuales"; por qué los varones homosexuales se involucraban en actividades sexuales durante sus viajes; qué actividades practicaban; $y$ con qué frecuencia utilizaban protección. Después de entrevistar a 94 turistas masculinos de origen alemán, el estudio concluyó que muchos viajeros visitaban el país con un propósito sexual,y que una amplia variedad de prácticas sexuales eran evidentes.

Más tarde, Clift y Forrest (1999) presentaron resultados de una encuesta a 562 hombres gay residentes del sur de Inglaterra, centrando su atención en la actividad sexual de estos varones durante los viajes. Se encontró que, cuando viajaban, aproximadamente la mitad de los encuestados tenía actividad sexual con algún compañero nuevo.Además se reveló que tenían mayores expectativas de estar sexualmente activos durante su viaje y de ser motivados por la vida social gay y por la actividad sexual. Sin embargo, como en otros casos (Ford et al., 1993), los resultados y las posibles generalizaciones derivadas de estudios precedentes deben ser considerados de manera crítica y cuidadosa, dadas las características y limitaciones específicas de cada investigación.

Publicaciones gay, turismo y sexo

Aunado a los resultados de la investigación académica, algunas publicaciones 
dirigidas a la comunidad gay indican que la actividad sexual es un motivador cardinal para algunos viajeros. Libros y guías turísticas tales como Spartacus Internacional Gay Guide (Bedford, 2005), por ejemplo, muestran imágenes y anuncios explícitamente sexuales que sin reserva promueven y recomiendan lugares en muchas partes del mundo donde son posibles encuentros sexuales casuales o comerciales.

De igual manera, Internet ha contribuido a difundir lugares propicios para encuentros sexuales entre viajeros gay. Páginas como www.cruisingforsex.com, la cual contiene información de más de cien países, detalla sitios en donde existen amplias posibilidades de actividad sexual gay (Luongo, 2002).Aunque la prensa turística dirigida al consumidor gay y las investigaciones realizadas hasta ahora muestran hechos, ideas generales, y hasta cierto punto sólo percepciones acerca de este tipo de turismo y su comportamiento sexual, es académicamente pertinente reconocer que ninguna conclusión significativa puede hacerse basándose en dichas fuentes. Debido a la naturaleza particular de los análisis y a la gran variedad en aspectos de muestreo y metodología, tiempos, escenarios, variables incluidas, objetivos de estudio, e incluso la posible subjetividad del investigador, los resultados deben tratarse con cuidado.

El presente estudio pretende subsanar la carencia investigativa respecto a las percepciones de las comunidades hacia el turismo gay y su comportamiento sexual en destinos turísticos, ignoradas en los estudios del sector (Hughes, 2006).

\section{Metodología}

En el contexto de estudios sobre turismo y sexo es evidente que una variedad de técnicas tanto cuantitativas como cualitativas han sido empleadas (Clift y Carter, 2000). Sin embargo, debido a las peculiaridades implícitas en las relaciones entre el turismo y el sexo, los académicos han optado casi siempre por el uso de técnicas cualitativas (Cohen, 1982; Dahles, 1998; Graburn, 1983). De hecho, se ha generalizado el punto de vista de que una consideración más cualitativa de las relaciones entre los viajes y el comportamiento sexual es esencial para comprender a profundidad su significado y las experiencias subjetivas que conllevan (Thomas, 2000); lo cual alude a las dimensiones émicas de dichas relaciones. La revisión teórica pone al descubierto que, atendiendo a esto, algunos investigadores han escudriñado los vínculos entre turismo y comportamiento sexual mediante entrevistas informales y a profundidad, 
observación participante y no participante, grupos de enfoque, y análisis de documentos personales (Cohen, 1982; Dahles, 1998; Leung, 2003; Phillip y Dann, 1998; Thomas, 2000).

Dada la naturaleza oculta de la población homosexual (Poria y Tailor, 200 I), en lo que respecta a estudios académicos sobre viajeros gay se recomienda el uso de métodos de investigación cualitativa para un mejor entendimiento de este tipo de fenómenos (Hughes, 2004; Pritchard et al., 2000), aunque algunos investigadores han optado por una aproximación cuantitativa (Clift y Forrest, 1999; Forrest y Clift, 1998).

Entre los trabajos que han recurrido a metodologías cualitativas, incluyendo entrevistas, observación participante y grupos de enfoque destacan: Ford et al., 1993; Herrera y Scott, 2005; Hughes, 2002; Luongo, 2000; Poria y Tailor, $200 \mathrm{I}$; Pritchard et al., 2000.

Finalmente en cuanto a las investigaciones acerca de las percepciones de residentes locales hacia el turismo, la mayoría de los estudios indican con nitidez una tendencia hacia el uso de aproximaciones cuantitativas, en su mayoría reflejadas en la aplicación de encuestas en comunidades receptoras. No obstante, algunos estudios han rescatado el valor émico de las metodologías cualitativas para abordar las percepciones hacia el turismo (Brunt y Courtney 1999;Spanou, 2007) y han demostrado que el uso de entrevistas a profundidad, así como grupos de enfoque merecen reconocimiento metodológico dentro del área de estudio.

\section{Escenario}

En términos generales, el estudio se realizó a nivel exploratorio en una playa nudista y su comunidad adyacente en la costa del Pacífico mexicano. Por cuestiones de estricta confidencialidad ofrecida a los informantes, el nombre y detalles de la localidad se omiten. Como destino turístico, a la playa concurren varios tipos de turistas, sobre todo extranjeros y un número considerablemente creciente de visitantes gay. Desde hace algunos años, debido a la demanda el sitio se ha identificado popularmente como uno de los pocos destinos gay en el país.

\section{Procedimiento}

Para los propósitos del presente estudio se optó por una aproximación cualitativa. Los datos fueron recolectados en diferentes periodos de 2004 a 2007. Se aplicaron doce entrevistas a residentes, empresarios de la comunidad 
adyacente y a visitantes gay. En cuanto a los dos primeros grupos, se incluyeron preguntas relativas a su percepción sobre turistas gay y el comportamiento sexual de éstos en los espacios públicos de la playa.A los visitantes gay se les interrogó específicamente sobre su estancia y las razones para visitar esa playa, la socialización y las actividades ordinarias; también se les preguntó acerca del uso de drogas y encuentros sexuales.

La mayoría de los informantes fueron reclutados a través de muestreo bola de nieve. Las entrevistas se realizaron en casa de los residentes, en la playa, restaurantes y hoteles; ocho fueron grabadas y transcritas para su análisis, del resto se tomaron notas relevantes.

Teniendo en cuenta las ventajas potenciales de la triangulación metodológica en investigaciones turísticas cualitativas (Decrop, 1999), se revisaron páginas web que contienen descripción de la localidad, y se registraron conversaciones informales con informantes. El análisis de contenido de páginas web y de las conversaciones sirvió para corroborar los datos previamente obtenidos. También se consultaron sitios web en donde viajeros y algunos residentes comparten ideas, experiencias y preocupaciones acerca de la playa. Debido a la gran variedad de temas existentes en esos sitios, se eligieron asuntos que de alguna manera se vinculaban con los objetivos del estudio.

\section{Resultados}

La información recabada fue muy amplia y abarcó varias dimensiones del objeto de estudio. No obstante, los resultados aquí reportados se limitan a algunos efectos que el turismo gay y su comportamiento sexual están teniendo en el destino. De igual manera, los resultados reportan ciertas preocupaciones que han surgido en la comunidad receptora.

\section{Presencia de visitantes gay y su comportamiento}

Del estudio se obtiene que la playa es visitada por varios tipos de turistas, tanto nacionales como internacionales, con un crecimiento notable de hombres gay que viajan sobre todo de Estados Unidos, Europa y otras ciudades de México. Fue muy frecuente oír en las entrevistas extractos como el siguiente: "Recuerdo que hace años era novedad ver uno (visitante gay) por acá. La gente se preguntaba acerca de ellos y los veían raro. ¡Ahora, los puedes ver en grupos de siete u ocho, lo juro!" 
Por lo que toca a su estancia, quedó de manifiesto que la mayoría de los turistas gay pasan la mayor parte del día en los extremos de la playa, conocidos en la localidad como espacios gay, lo cual coincide con modelos geográficos previamente propuestos (Jaurand y de Luze, 2004). Se reveló que estos lugares son frecuentados en su mayoría por turistas gay que consideran dichas áreas como lugares comunes para socializar, "ligar" y tener encuentros sexuales, lo que también concuerda con investigaciones incipientes (Douglas et al., 1977) y, a su vez, recientes (Schoemaker, 2006).

En cuanto a la actividad sexual gay, fue difícil estimar la incidencia precisa de turistas gay involucrados en encuentros sexuales en el destino. Sin embargo, informantes reportaron: a) que con cierta frecuencia existe actividad sexual gay al aire libre en la playa; b) que los encuentros sexuales entre individuos de la misma edad son tan comunes como entre individuos de diferente edad; c) que los encuentros sexuales son ligeramente más comunes entre individuos de diferentes nacionalidades, y d) que la falta de habilidades lingüísticas no es un obstáculo para la actividad sexual casual. Según los entrevistados, actos sexuales como exhibicionismo, voyeurismo, masturbación y felación tienen lugar en espacios públicos de la playa.

Investigador: ¿Alguna vez ha sabido, visto o escuchado acerca de encuentros sexuales homosexuales en el destino turístico?

Empresario: He vivido a un lado de la playa [lado este], en la colina, por los últimos diez años. Eso lo veo muy frecuentemente, especialmente durante diciembre y Semana Santa. Los he visto uno al lado de otro, abrazándose, teniendo sexo oral... y a veces no son sólo dos, sino más de dos...

El comentario de un visitante reportó:

Hace unas semanas, hubo una fiesta gay en el este de la playa en la noche, pero no asistí porque algunas personas me dijeron que eso era una orgía y yo no le hago a eso.

Un visitante gay de 26 años refirió:

He visitado el lugar cuatro veces... No tolero a los gay mayores. No me gustan... Creo que sólo acosan todo el tiempo. Siempre están detrás de chicos jóvenes.A veces se acercan y te invitan a tener sexo. Este lado de la 
playa [lado oeste] está ahora asediada por adultos gay, adultos que sólo están al asecho de jóvenes... yo incluso los llamo "escualos" porque siempre están detrás de una presa; de una presa joven.

\section{Beneficios percibidos y otros aspectos}

En relación con las repercusiones específicas, las entrevistas mostraron que el impacto económico positivo del turismo gay en la localidad es muy reconocido, en particular por propietarios de negocios.

Investigador: ¿Piensa usted que el turismo gay tiene efectos positivos en la comunidad?

Informante: ...Sí, después de todo, los homosexuales son los que poseen buen dinero. No tienen responsabilidades de familia, así que tienen suficiente para gastar. Ellos pueden pasar una semana o más, a ellos no les importa pagar mucho y lo hacen para sentirse cómodos. Es un tipo de turismo que no le importa gastar su dinero... se preocupa por verse bien, estar bien y disfrutar. El turista gay sabe cómo disfrutar y si tiene el dinero para hacerlo, no le importa gastarlo...; económicamente hablando, el turista gay es un buen turista en realidad.

Otros informantes mantienen "percepciones positivas" del turismo gay. Por ejemplo, un propietario de hospedaje que ha vivido en la localidad por más de 20 años comentó:

Los visitantes gay tienen efectos positivos como cualquier otro turista, creo. Pero tenemos que admitir que también son personas amables, bien educadas, cultas y, lo más importante para algunos de nosotros, ellos sí tienen dinero.

Durante el trabajo de campo se encontraron aspectos relevantes en cuanto a la movilidad de estos turistas. Sin duda, uno de ellos es que el lugar está siendo rápidamente identificado como un lugar popular entre turistas gay. La migración de visitantes gay ha jugado un papel crucial en esto. Mientras que la mayoría de los encuentros sexuales son de naturaleza temporal, algunos individuos han recurrido a relaciones formales. Como consecuencia, parejas gay biculturales (dos casos reportados) en las cuales uno de los miembros ha 
abandonado de forma definitiva su país para por emigrar a la comunidad. Después de establecerse, buscan empleo, o incluso inician su propio negocio en el destino turístico.

\section{Costos percibidos}

Aunado a lo anterior y acerca de los efectos del comportamiento sexual del turismo gay en el destino, los locales expresaron algunas preocupaciones, por ejemplo la alienación de otros tipos de turismo y de la comunidad local misma, la prostitución y el uso de drogas, entre los más citados.

Como se mencionó antes, además de visitantes gay, otro tipo de turistas y residentes locales visitan la playa. En este escenario, el comportamiento sexual público de los gay en la playa parece estar ahuyentando otras formas de turismo, lo cual, se presume, significa pérdidas económicas para algunos empresarios locales.

Empresario: ...es una mala situación porque a esos visitantes [gay] simplemente no les importa; sólo quieren tener sexo... Hace unos meses, hubo una pareja, un hombre y una mujer, que visitaban el lado oeste de la playa. En cuanto llegaron, partieron inmediatamente, regresaron al hotel y dijeron:"no podemos estar aquí por lo que está sucediendo en la playa. Sólo hay gays y están haciendo sus porquerías". Como empresario, estoy empezando a perder negocio porque a la gente heterosexual no le gusta ese tipo de ambiente, y difícilmente regresarán, y no los culpo; el sexo debe ser privado, no debe ser público, y no debes hacer eso frente a perfectos extraños; especialmente si no son de tu misma orientación sexual.

Con respecto a la alienación de las familias locales, un ama de casa dijo:

Aquí, mucho nos ha afectado el nudismo gay... Hay familias que ya no pueden visitar la playa. No es sólo el hecho de que estén desnudos [visitantes gay]; han llegado al extremo de estar haciendo cosas, que ya no podemos visitar la playa... Yo misma los he visto, en parejas, dos hombres haciendo sus cosas sexuales en la playa a la luz del día. Para mí, fue horrible.

En el contexto descrito, y pese a que los datos disponibles son limitados por tratarse de un fenómeno que procura ocultarse, la prostitución masculina 
fue reportada como otro asunto relevante. La información evidenció que jóvenes de localidades aledañas -que se ubican a lo largo de la playa o en la disco local- están involucrados en prostitución; no necesariamente se trata de jóvenes gay, pero ofrecen servicios sexuales si son remunerados por ello $y$, aunque se reporta que la mayoría de ellos son mayores de edad, según elementos de análisis de contenido de sitios web, hay indicios de que también participan en la prostitución menores de edad.

Con los datos obtenidos se puso al descubierto que el uso de drogas ha sido siempre común no sólo entre los turistas sino también entre algunos miembros de la comunidad local. En algún momento, a todos los visitantes entrevistados les fueron ofrecidas drogas. Respecto a los visitantes gay con los que hablamos, algunos aceptaron abiertamente el haberlas consumido durante su estancia; sin embargo, la conexión entre visitantes gay y el consumo de drogas alcanza otras dimensiones. El trabajo de campo reveló que el uso de drogas entre "prostitutos" está siendo motivado por turistas gay debido a que los "prostitutos" pueden solicitar drogas en lugar de dinero a cambio de sexo.

Investigador: ¿Ha presenciado algún caso en que se haya ofrecido o vendido sexo en la localidad?

Visitante gay nacional: Sí, yo mismo lo he experimentado. El contacto usualmente tiene lugar en la playa o en la disco. Usualmente son chicos que vienen de los alrededores. Vienen por dinero o drogas. Ellos saben cómo está la situación aquí y saben cómo atraerte... Cuando te miran, puedes pensar que les gustas. Después te invitan a caminar en la playa. Una vez en la playa, de noche, te ofrecen sexo por 200 o 300 pesos. Yo he sabido de gente que ha pagado por eso. También he sabido de otros que permiten tener sexo oral sólo por algo de marihuana.

Un visitante gay norteamericano narró su propia experiencia:

Yo estaba en mi hotel en la noche, casi listo para dormir cuando este chico joven, creo que era local, tocó a mi puerta. Me dijo que se le había acabado el repelente para mosquitos y me pidió prestado el mío. Le di un poco y comenzamos a platicar... Después me dijo que le prestara unos cuantos dólares y que me pagaría con sexo en mi habitación esa misma noche. 
Además de los resultados relacionados con el aspecto sexual del turismo gay en la comunidad, la naturaleza exploratoria y cualitativa del estudio permitió observar que otros fenómenos de interés tanto académico como social están teniendo lugar en la comunidad receptora. La inmigración indeseada de, en palabras de residentes locales, "malandrines extranjeros", fue revelada por el estudio. Asimismo, cuestiones relacionadas con el comportamiento de otro tipo de visitantes -nudistas-resultaron de relevancia en términos de investigación académica. Sin embargo, debido a los objetivos del artículo, el análisis a profundidad de estos últimos resultados escapa a los alcances aquí presentados.

\section{Conclusiones}

Además de pretender contribuir al entendimiento conceptual y teórico de las percepciones de comunidades anfitrionas hacia el turismo gay, su comportamiento sexual y sus impactos en ellas, el estudio tuvo como objetivo reconocer las ventajas del uso de aproximaciones cualitativas para temas delicados dentro de la investigación turística. Con base en los resultados obtenidos mediante la combinación de entrevistas, análisis de contenido de páginas web y análisis de conversaciones informales, la triangulación metodológica para estudios turísticos resultó altamente valorada como una alternativa para validar datos en investigaciones semejantes.

Este trabajo es uno de los primeros en investigar las percepciones de residentes locales hacia el comportamiento sexual del turismo gay y sus repercusiones en un país en vías de desarrollo. Aunque la comunidad anfitriona reconoce como un elemento positivo la aportación económica del turismo gay, también percibe que ciertas formas de comportamiento sexual están contribuyendo al desarrollo de problemas sociales tales como la prostitución varonil y el uso de drogas entre los miembros de comunidades locales. Además de que el comportamiento sexual del turismo gay está alienando del destino a otras formas de turismo y a la comunidad local misma.

Conforme a las proposiciones deWall y Mathieson (2006), es indispensable considerar los efectos del turismo en comunidades receptoras para poder asegurar que los objetivos de la sustentabilidad social sean alcanzados. Por ello, es importante que las autoridades de planeación y gestión turística-locales, regionales, nacionales, e incluso internacionales-, evalúen las consecuencias 
que potencialmente tengan en comunidades receptoras vulnerables ciertas formas de comportamiento sexual del turismo.

Pese a que esta investigación puede proveer una idea al respecto, inevitablemente, como en cualquier otro estudio, existen limitaciones que no deben ignorarse: los resultados están acotados a un destino turístico pequeño con particularidades específicas; asimismo, no se puede definir con claridad qué impactos exclusivamente pertenecen al turismo y cuáles a una combinación de éste y del contexto social, cultural y económico inherente a la localidad objeto de estudio. Por ello, se requiere mayor investigación para establecer hasta qué grado los resultados aquí ofrecidos coinciden o difieren de aquellos en destinos con otras características. El uso de una aproximación metodológica distinta podría también ser de utilidad para comparar o contrastar los resultados interpretativos descritos.

\section{FUENTES DE CONSULTA}

Agrusa, J. F. (2003). “AIDS and tourism: A deadly combination”, en T. Bauer y B. McKercher. Sex and tourism:Journeys of romance, love and lust. Londres: The Haworth Hospitality Press (THHP), 167-I80.

Beddoe, C. (2003). "Ending child sex tourism: A vision for the future", en T. Bauer y B. McKercher. Sex and tourism: Journeys of romance, love and lust. Londres: THHP, 197-207.

Bedford, B. (2005). Spartacus International Gay Guide (32 ed.). Berlín: Bruno Gmunder.

Brunt, P. y P. Courtney (1999). "Host perceptions of socio-cultural impacts”. Annals of Tourism Research, 26, 3, 493-5I 5.

Clift, S. y S. Carter (2000). Tourism and sex: Culture, commerce and coercion. Londres: Pinter.

(1999)."Gay men and tourism: Destinations and holiday motivations”. Tourism Management, 20, 615-625.

y J.Wilkins (1995). “Travel, sexual behaviour and gay men”, en P.Aggleton, P. Davies y G. Hart. AIDS: Safety, sexuality and risk. Londres:Taylor and Francis, 35-54.

y S. Forrest (1999).“Factors associated with gay men's sexual behaviours and risk on holiday". AIDS Care, I I, 3, 28I-295. 
Cohen, E. (1982). "Thai girls and farang men:The edge of ambiguity”. Annals of Tourism Research, 9, 403-428.

Dahles, H. (1998). "Of birds and fish: Street guides, tourists, and sexual encounters in Yogyakarta, Indonesia", en M. Oppermann. Sex tourism and prostitution:Aspects of leisure, recreation, and work. Nueva York: Cognizant Communication Corporation, 30-40.

Decrop,A. (I999). “Triangulation in qualitative tourism research”. Tourism Management, 20, I57-I6I.

Douglas, J., P. Rasmussen y C. Flanagan (1977). The nude beach. California: Sage Publications.

Ford, K., D. N.Wirawan y P. Fajans (I 993)."AIDs knowledge, condom beliefs and sexual behaviour among male sex workers and male tourist clients in Bali, Indonesia”. Health Transition Review, 3, 2, I 91-204.

Forrest, S. y S. Clift (1998). "Gay tourist space and sexual risk behaviour”, en C.Aitchinson y F. Jordan. Gender, space and identity: Leisure, culture and commerce. Eastbourne: Leisure Studies Association, I63-I76.

Graburn, N. H. ( 1983 ). “Tourism and prostitution”. Annals of Tourism Research, 10, 437-456.

Herrera, S. L. y D. Scott (2005). “'We gotta get out of this place!' Leisure travel among gay men living in a small city". Tourism Review International, 8, 249-262.

Hobson, J. S. y U. C. Dietrich ( 1994). “Tourism, health, and quality of life: Challenging the responsibility of using the traditional tenets of sun, sea, sand, and sex in tourism marketing". Journal of Travel \& Tourism Marketing, 3, 4, 21 -38.

Hughes, H. (2002). “Gay men's holiday destination choice: A case of risk and avoidance". International Journal of Travel Research, 4, 299-3 I 2.

(2004). "A gay tourism market: Reality or illusion, benefit or burden?" Journal of Quality Assurance in Hospitality \& Tourism, 5, 2-4, 57-74.

- (2006). Pink tourism: Holidays of gay men and lesbians. Oxfordshire: $\mathrm{CABI}$ Publishing.

Jaurand, E.y H. de Luze (2004).“Ces plages où es genres s'affichent? Les territoires du nu sur la Côte d'Azur”, en C. Bard. Le genre des territoires: Masculin, féminin, neutre. Angers: Presses de l'Université d'Angers, 227-240.

Jeffreys, S. (2003). "Sex tourism: Do women do it too?" Leisure Studies, 22, 223-238. 
Josiam, B. M., et al. (1998). "An analysis of the sexual, alcohol and drug related behavioural patterns of students on spring break". Tourism Management, I9, 6, 50I-5I3.

Kempadoo, K. (1999). Sun, sex and gold:Tourism and sex work in the Caribbean.

Oxford: Rowman \& Littlefield.

Leung, P. (2003). "Sex tourism:The case of Cambodia", en T. Bauer y B. McKercher. Sex and tourism: Journeys of romance, love and lust. Londres: THHP, $18 \mid-195$

Luongo, M. (2000). "The use of commercial sex venues and male escorts by gay tourists in New York city", en S. Clift y S. Carter. Tourism and sex: Culture, commerce and coercion. Londres: Pinter, I07-I30.

(2002). “Appendix: Gay and lesbian travel resources guide”, en S. Clift, M. Luongo y C. Callister. Gay tourism: Culture, identity and sex. Londres: Continuum, 267-276.

McKercher, B.yT. Bauer (2003)."Conceptual framework of the nexus between tourism, romance, and sex”, en T. Bauer y B. McKercher. Sex and tourism: Journeys of romance, love and lust. Londres: THHP, 3-I7.

Nyanzi, S., et al. (2005)."Bumsters, big black organs and old white gold: Embodied racial myths in sexual relationships of Gambian beach boys". Culture, Health \& Sexuality, 7, 6, 557-569.

Oppermann, M. (1999). “Sex tourism”. Annals of Tourism Research, 26, 2, 25I-266. Phillip,J.y G. Dann (1998). "Bar Girls in Central Bangkok: Prostitution as Entrepreneurship”, en M. Oppermann. Sex tourism and prostitution:Aspects of leisure, recreation and work. Nueva York: Cognizant Communication Corporation, 60-70.

Poria, Y. y A. Tailor (200I). “'I am not afraid to be gay when I'm on the net': Minimising social risk for lesbian and gay consumers when using the Internet". Journal of Travel and Tourism Marketing, I I, 2-3, I27-I 42.

Pritchard, A., et al. (2000). "Sexuality and holiday choices: Conversations with gay and lesbian tourists". Leisure Studies, 19, 267-282.

Rogstad, K. E. (2004). "Sex, sun, sea, and STIs: Sexually transmitted infections acquired on holiday". BMJ, 329, 2। 4-2 I7.

Schoemaker,J. (2006)."Bare bodies, beaches, and boundaries:Abjected outsiders and rearticulation at the nude beach". Sexuality \& Culture, 10, 4, 29-53.

Seabrook, J. (200 I). Travels in the skin trade ( $2^{\mathrm{a}}$ ed.). Londres: Pluto Press. 
Comunidades receptoras y percepciones: un estudio sobre turismo y sexualidad

Spanou, E. (2007). "The impact of tourism on the sociocultural structure of Cyprus". Tourismos:An International Multidisciplinary Journal of Tourism, 2, I, I45-162.

Thomas, M. (2000)."Exploring the contexts and meanings of women's experiences of sexual intercourse on holiday”, en S. Clift y S. Carter. Tourism and sex. Londres: Pinter, 200-220.

Wall, G.y A. Mathieson (2006). Tourism: Change, impacts and opportunities. Essex: Pearson Prentice Hall. 